This is the peer reviewed version of the article: "Burden of disease from exposure to second-hand smoke in children in Europe", which has been published in final form at https://doi.org/10.1038/s41390-020-01223-6 with reference:

Carreras, G., Lachi, A., Cortini, B. et al. Burden of disease from exposure to secondhand smoke in children in Europe. Pediatr Res 90, 216-222 (2021).

\title{
Burden of disease from exposure to second-hand smoke in children in Europe
}

Giulia Carreras ${ }^{1 *}$, Alessio Lachi ${ }^{1}$, Barbara Cortini $^{1}$, Silvano Gallus $^{2}$, Maria José López ${ }^{3,4,5}$, Ángel López Nicolás ${ }^{6}$, Alessandra Lugo ${ }^{2}$, Maria Teresa Pastor ${ }^{7}$, Joan B Soriano ${ }^{7,8}$, Esteve Fernandez $z^{8,9,10,11}$, Giuseppe Gorini ${ }^{1}$, and TackSHS Project Investigators ${ }^{12}$.

\section{Author contribution:}

GC, AL, BC, SG, EF, GG made substantial contributions to conception and design, acquisition of data, or analysis and interpretation of data;

MJL, ALN, AL, MTP, JBS, EF contributed in drafting the article or revising it critically for important intellectual content;

GC, AL, BC, SG, MJL, ALN, AL, MTP, JBS, EF, GG gave the final approval of the version to be published.

\section{Authors affiliation:}

${ }^{1}$ Oncologic network, prevention and research institute (ISPRO), Florence, Italy

2 Istituto di Ricerche Farmacologiche Mario Negri IRCCS (IRFMN), Milan, Italy

${ }^{3}$ Public Health Agency of Barcelona (ASPB), Barcelona, Spain.

${ }^{4}$ CIBER Epidemiologia y Salud Pública (CIBERESP), Barcelona, Spain

${ }^{5}$ IIB Sant Pau, Barcelona, Spain

${ }^{6}$ Polytechnic University of Cartagena (UPCT), Cartagena, Spain

${ }^{7}$ Hospital Universitario La Princesa (IISP), Madrid, Spain 
${ }^{8}$ Consortium for Biomedical Research in Respiratory Diseases (CIBER en Enfermedades Respiratorias, CIBERES), Madrid, Spain

${ }^{9}$ Catalan Institute of Oncology (ICO), L’Hopitalet de Llobregat, Spain

${ }^{10}$ Bellvitge Biomedical Research Institute (IDIBELL), L'Hopitalet de Llobregat, Spain

${ }^{11}$ University of Barcelona, Barcelona, Spain

12 The TackSHS Project Investigators:

Catalan Institute of Oncology (ICO); Bellvitge Biomedical Research Institute

(IDIBELL), Spain: Esteve Fernández, Yolanda Castellano, Marcela Fu, Montse Ballbè, Beladenta Amalia, Olena Tigova

Public Health Agency of Barcelona (ASPB), Spain: Maria José López, Xavier Continente, Teresa Arechavala, Elisabet Henderson

Istituto di Ricerche Farmacologiche Mario Negri IRCCS (IRFMN), Italy: Silvano Gallus, Alessandra Lugo, Xiaoqiu Liu, Elisa Borroni; Istituto DOXA, Worldwide Independent Network/Gallup International Association, Italy: Paolo Colombo University of Stirling (UNISTIR), the UK: Sean Semple, Rachel O'Donnell, Ruaraidh Dobson

TobaccoFree Research Institute Ireland (TFRI), Ireland: Luke Clancy, Sheila Keogan, Hannah Byrne

Hellenic Cancer Society - George D. Behrakis Research Lab (HCS), Greece: Panagiotis Behrakis, Anna Tzortzi, Constantine Vardavas, Vergina Konstantina Vyzikidou, Gerasimos Bakelas, George Mattiampa

Fondazione IRCCS Istituto Nazionale dei Tumori (INT), Italy: Roberto Boffi, Ario Ruprecht, Cinzia De Marco, Alessandro Borgini, Chiara Veronese, Martina Bertoldi, Andrea Tittarelli 
Istituto per lo Studio, la Prevenzione, e la Rete Oncologica (ISPRO), Italy: Giuseppe Gorini, Giulia Carreras, Barbara Cortini, Simona Verdi, Alessio Lachi, Elisabetta Chellini

Polytechnic University of Cartagena (UPCT), Spain: Ángel López Nicolás, Marta TraperoBertran, Daniel Celdrán Guerrero

European Network on Smoking and Tobacco Prevention (ENSP), Belgium: Cornel RaduLoghin, Dominick Nguyen, Polina Starchenko

Hospital Universitario La Princesa (IISP), Spain: Joan B Soriano, Julio Ancochea, Tamara Alonso, María Teresa Pastor, Marta Erro, Ana Roca, Patricia Pérez, Elena García Castillo

\section{* Correspondence:}

Giulia Carreras

Oncologic network, prevention and research institute (ISPRO)

via Cosimo il Vecchio, 2 - 50139 Florence, Italy

phone: +39 0554223846731

e-mail: g.carreras@ispro.toscana.it

\section{Statement of financial support:}

This work was supported by the European Union's Horizon 2020 research and innovation programme [grant number 681040]; the 2017 Italian Health Ministry CCM Programme Project "Choosing health priorities and selecting effective interventions to prevent the burden of chronic non-communicable diseases" (http://www.ccmnetwork.it/progetto.jsp?id=node/1977\&idP=740); the Ministry of Research and Universities from the Government of Catalonia to EF [grant number 2017SGR319]; the Instituto Carlos III and co-funded by the European Regional Development Fund, FEDER [INT16/00211 and 
INT17/00103], Government of Spain to EF and the Italian League Against Cancer (Milan) to SG.

\section{Disclosure statement:}

This manuscript was prepared by the TackSHS Project Consortium and does not necessarily reflect the views of the European Commission. The European Commission is not responsible for any use that may be made of the information that contains in this manuscript.

All Authors have no competing interests to declare.

Patient consent statement: patient consent was not required.

Category of study: Clinical Research Article

\section{Impact Statement:}

- Exposure to second-hand smoke at home and in pregnancy is a major cause of disease among children.

- Smoking legislation produced the adoption of voluntary smoking bans in homes, however second-hand smoke exposure at homes still occurs and its burden is substantial.

- In 2017 the number of deaths and disability-adjusted life years in children attributable to exposure to second-hand smoke in the European Union countries were respectively 335 and 35633 .

- Low birth weight caused by second-hand smoke exposure in pregnancy showed the largest burden.

- Eastern European Union countries showed the highest burden. 


\begin{abstract}
Background

Second-hand smoke (SHS) exposure at home and fetal SHS exposure during pregnancy are a major cause of disease among children. The aim of this study is quantifying the burden of disease due to SHS exposure in children and in pregnancy in 2006-2017 for the 28 European Union (EU) countries.

Methods

Exposure to SHS was estimated using a multiple imputation procedure based on the Eurobarometer surveys, and SHS exposure burden was estimated with the Comparative Risk Assessment method using meta-analytical relative risks. Data on deaths and disabilityadjusted life years (DALY) were collected from National statistics and from the Global Burden of Disease Study.

Results
\end{abstract}

Exposure to SHS and its attributable burden stalled in 2006-2017; in pregnant women, SHS exposure was $19.8 \%$ in $2006,19.1 \%$ in $2010,21.0 \%$ in 2017 ; in children it was $10.1 \%$ in $2006,9.6 \%$ in $2010,12.1 \%$ in 2017 . In $2017,35,633$ DALYs among children were attributable to SHS exposure in the EU, mainly due to low birth weight.

Conclusions

Comprehensive smoking bans up to 2010 contributed to reduce SHS exposure and its burden in children immediately after their implementation, however SHS exposure still occurs and, in 2017, its burden in children was still relevant. 


\section{Introduction}

Exposure to second-hand smoke (SHS) is a major cause of disease among children, and there is no safe level of SHS exposure (1-2). It has been shown to be associated with many adverse health effects among children, and the diseases with the strongest evidence of a causal link with SHS exposure in children are asthma, lower respiratory tract infections (LRI), otitis media (OM), and sudden infant death syndrome (SIDS)(2-3). Moreover, exposure to SHS during pregnancy are detrimental to fetal growth and development, leading to adverse birth outcomes such as preterm birth, low birthweight (LBW), being small for gestational age, and perinatal and infant mortality (2-3).

Children are involuntarily exposed to SHS from their parents smoking at home. However, in the last 20 years in many countries worldwide, the implementation of smoking bans in workplaces, including the hospitality sector, following Article 8 of the World Health Organization (WHO) Framework Convention for Tobacco Control, have had, in the early years, an indirect effect of increasing voluntary smoking bans in homes, that may also result from increases in cessation rates in household members (4-6). For example, smoke-free homes increased from $72 \%$ in 2008 to $78 \%$ in 2012 in Italy (7-8), from $16 \%$ in 1998 to almost $50 \%$ in 2008 in smokers' houses in England (9), by 50\% in Scotland (10), and SHS exposure at home declined from $29.2 \%$ in 2006 to $12.7 \%$ in 2011 in Spain (11). Moreover, the introduction of smoke-free legislation in Scotland showed a 39\% reduction in salivary cotinine concentration among children (12).

As a consequence of the adoption of smoke-free houses, smoking bans had the further indirect effect of reducing the diseases in children due to SHS exposure mainly at home, and in newborns, due to SHS exposure among pregnant women in any setting. A recent metaanalysis that included 41 studies, among which 16 from Europe, showed an association between the implementation of smoke-free legislation and reductions in the rates of 
hospitalization from asthma exacerbations $(-9.8 \%, 95 \%$ confidence intervals $[\mathrm{CI}]:-16.6 \%$,3.0\%), LRI (-18.5\%, 95\%CI: $-32.8 \%,-4.2 \%)$, and LBW $(-2.8 \%, 95 \% \mathrm{CI}:-4.4 \%,-1.2 \%)$ in children (13). In Scotland, smoke-free legislation was associated with a gradual decrease in acute LRI events (14), and in the rate of asthma admissions in children (15). In Hungary, the strengthening of smoking bans improved the probability of live births (16), and the introduction of national smoke-free legislation in England was associated with fewer hospital admissions per year for respiratory tract infection and asthma in children (17-18).

Despite the increase of smoke-free homes in the early years after smoking legislation with subsequent increase in children's health, many children and pregnant women are still exposed to SHS in European Union (EU) countries in recent years. As an example, in 2010 in Italy, $54 \%$ of youths were exposed to SHS in any setting (19), and in 2016, $72 \%$ of children under 12 years were exposed in any setting in Spain (20). Moreover, in a Spanish birth cohort, 30\% pregnant women were exposed to SHS during 1997 to 2008 (21), in Poland the prevalence in pregnant women was estimated as $13 \%$ in 2008 (22), and, recently, in a sample of Slovak pregnant women $28 \%$ of them lived with a smoking partner (23).

As a consequence, the burden of disease due to SHS exposure in children should be still considerable.

A full understanding of the exposure to SHS at home in children and the consequent burden of diseases for the whole EU population of children and newborns in the last decade is lacking. The aim of this study, conducted within the TackSHS project (24), is to quantify the burden of disease due to SHS exposure at home among children and to SHS exposure in pregnancy in the last 10 years in $28 \mathrm{EU}$ countries.

\section{Methods}

Burden of disease from second-hand exposure 
The burden of disease from exposure to SHS in children was estimated for each country using the comparative risk assessment methodology (25). In brief, we selected the diseases for which there is evidence of a causal relationship with SHS exposure and for which there is sufficient quantitative information (25). Then for each SHS-related disease (LBW, SIDS, LRI, OM and asthma exacerbation) the country-specific mortality and morbidity attributable to exposure to SHS was calculated by first estimating the SHS attributable fraction $\left(\mathrm{AF}_{\mathrm{SHS}}\right)$ for each age-group using the Levin's formula (26)

$A F_{S H S}=\frac{p \cdot(R R-1)}{p \cdot(R R-1)+1}$

where $p$ is the prevalence of exposure to SHS (by age groups in each country) and $R R$ is the corresponding relative risk of death or disease from the SHS-related disease for exposed to SHS compared to not exposed.

For each disease and country, the population $\mathrm{AF}_{\mathrm{SHS}}(\mathrm{PAF})$ was then obtained as a weighted sum of age-specific $\mathrm{AF}_{\mathrm{SHS}}$ with the proportion of cases in each stratum as weight. The PAF represents the reduction in the population burden of disease that would occur if exposure to SHS was removed. The age-groups included in the analysis varied depending on the disease under study. The number of deaths attributable to SHS exposure was obtained by multiplying the age-specific number of deaths for the corresponding $\mathrm{AF}_{\mathrm{SHS}}$ and summing over ages. The number of disability-adjusted life years (DALYs) was similarly computed.

For each estimate of deaths and DALYs attributable to SHS exposure, an uncertainty interval (UI) was obtained with a Monte Carlo procedure which deal simultaneously with uncertainty arising from estimates of SHS exposure, RR and deaths or DALYs (25) (see Supplementary material).

Exposure to second-hand smoke 
We used data from the Eurobarometer surveys carried out in 2006, 2010, 2014 and 2017, that are regular face-to-face interviews with approximately 1,000 subjects in each EU country (see Supplementary Material). We excluded the 2008 and 2012 surveys in order to contain the amount of data (and missing data) and to maintain a 3-4 year interval between surveys. Since SHS exposure was not uniformly asked in all surveys we used a multiple imputation procedure to estimate SHS exposure at home for the missing years (see Supplementary Material) (27).

In detail, we created a new data set including socio-demographic, smoking and SHS exposure variables from all the surveys (28), and we replaced each missing value with a plausible one $M$ times (creating $M$ complete datasets), choosing $M$ according to the rule of thumb based on the average percentage rate of missingness (29). This step was carried out with the Multivariate Imputation by Chained Equations procedure which involves specifying a posterior distribution for all incomplete variables and required a preliminary step consisting in the selection of a set of predictors to be included in the regressions $(27,30)$. The predictors included socio-demographic individual variables, such as gender, age, marital status, and survey's specific variables, such as country, year and survey weight. The regressions used varied according to the nature of the variables (logistic or polytomous logistic regressions and proportional odds models). Each of the $M$ complete datasets was then analyzed with standard statistical methods and the results were then pooled to obtain a final point estimate and a measure of precision which accounts for uncertainty due to missing information (27). Our data presented two different patterns of missing values. The first one is systematically missing data, which are those variables completely missing in some surveys. The second type of missing values, i.e. sporadically missing data, is partly and randomly missing in some surveys. We took into account for the clustered structure of the data coming from different surveys by forcing the survey year to be a predictor $(28,31)$. 
The final SHS exposure for children used in the burden estimation represents the proportion of people with children that allow smoking in their house and were specific for age groups 0-9 and 10-14 years. Exposure among pregnant women was defined as the proportion of women aged 25-44 years being ever exposed to SHS in any setting (house, workplace, car or public places). The age class 25-44 was chosen in order to take into account for the average age of women at childbirth in EU countries, that, in 2017, varied between 26 years of age in Bulgaria and 31 years of age in Italy, with an average of 29 years in EU (32). Both SHS exposure measures were estimated taking into account for the survey weights ensuring that each country is represented in proportion to its population size and to its sample composition (see Supplementary material).

\section{Data}

The RRs of death or incidence from LBW, SIDS, LRI, OM and asthma exacerbation for exposed to SHS in comparison to not exposed were estimated from the most recent metaanalyses. In table 1 the selected RRs values, with the referring age-groups, SHS exposure definition and references are reported.

Table 1 - Secondhand smoke (SHS) exposure related diseases, relative risks (RR) and corresponding 95\% confidence intervals (CI) estimate with their reference and SHS exposure definition in children.

\begin{tabular}{|l|l|l|l|l|}
\hline Disease & Age (years) & RR (95\% CI) & Reference & SHS exposure \\
\hline Low birth weight & 0 & $1.3(1.1-1.6)$ & $(33)$ & retrospectively assessed \\
maternal domestic, \\
Sudden infant
\end{tabular}




\begin{tabular}{|l|l|l|l|l|}
\hline \multirow{2}{*}{$\begin{array}{l}\text { Lower respiratory } \\
\text { infections }\end{array}$} & $<2$ & $1.5(1.4-1.7)$ & $(35)$ & $\begin{array}{l}\text { any household member } \\
\text { smoking }\end{array}$ \\
\cline { 2 - 5 } & $3-6$ & $1.8(1.1-1.2)$ & $(35-36)$ & $\begin{array}{l}\text { any household member } \\
\text { smoking }\end{array}$ \\
\hline Asthma & $<14$ & $1.3(1.2-1.4)$ & $(37)$ & either parent \\
\hline Otitis media & $<4$ & $1.3(1.2-1.5)$ & $(38)$ & household smoking \\
\hline
\end{tabular}

For years 2006, 2010 and 2014, the age-specific number of deaths from each disease for each country were extracted from the Eurostat (http://ec.europa.eu/eurostat/data/database/) and WHO (http://data.euro.who.int/dmdb/) databases that report observed data from EU Members States' National Statistical Institutes. For year 2017 death were extracted from the Global Health Data Exchange of the Global Burden of Disease (GBD) study, an international project that annually quantifies and globally compares the health loss due to diseases, accidents and risk factors by age, sex and geographical area and is compliant with the Guidelines for Accurate and Transparent Health Estimates Reporting (39-40).

For years 2006, 2010 and 2014 the country- and age-specific DALY from each disease were computed by first estimating the years of life lost (by applying the life expectancy extracted from Eurostat to the number of deaths collected from the Eurostat/WHO databases) and then summing them to the years lived with disability estimated within the GBD study. For 2017 the DALY estimates of GBD were used.

In Supplemental Table S4 of the Supplementary Material the list of ICD X codes used to select the diseases from the $\mathrm{WHO} /$ Eurostat databases and from the GBD study are presented. While the same classification was used to identify deaths and DALYs from SIDS and asthma from WHO/Eurostat and GBD, some differences were observed in the codes for LBW, LRI and OM (see Supplementary Material). 


\section{Results}

The multiple imputation procedure was run with $M=80$ imputations. Exposure to SHS at home in the overall EU among children stalled from 2006 to 2017 with values of $10.1 \%$ in 2006 (95\% confidence interval [CI]: 4.1\%-16.1\%), 9.6\% (95\%CI: 1.8\%-17.9\%) in 2010, $10.8 \%(95 \% \mathrm{CI}: 1.9 \%-20.2 \%)$ in 2014 , and $12.1 \%$ (95\%CI: $3.3 \%-21.4 \%)$ in 2017 . Exposure to SHS for pregnant women in the overall EU stalled from 2006 to 2017 with values of $19.8 \%$ in 2006 (95\% CI: 12.6\%-27.1\%), 19.1\% (95\%CI: 8.8\%-29.4\%) in 2010, 20.4\% (95\%CI: $9.2 \%-$ $31.7 \%$ ) in 2014, and 21.0\% (95\%CI: 8.7\%-33.4\%) in 2017. Prevalence estimates were largely uncertain due to the large amount of missing data taken into account in the multiple imputation procedure, and by visual inspection of confidence intervals, we can conclude that differences in prevalence were not significant.

As a consequence of the trend in SHS prevalence, the SHS-PAF in the EU countries were stables for all the diseases (table 2).

Table 2 - Secondhand smoke (SHS) exposure population attributable fractions (average among EU countries) by cause.

\begin{tabular}{rccccc}
\hline & \multicolumn{5}{c}{ Cause, Percentage (credibility interval) } \\
\cline { 2 - 6 } Calendar year & Otitis media & weight & Asthma & infections & deaths syndrome \\
\hline 2006 & $2.9(1.8-4.5)$ & $5.7(1.3-11.7)$ & $3.0(1.9-4.6)$ & $4.8(3.0-7.3)$ & $4.0(0.6-9.6)$ \\
2010 & $2.8(1.6-4.7)$ & $5.4(1.1-12.2)$ & $2.8(1.4-5.0)$ & $4.6(2.4-7.9)$ & $3.5(0.5-9.9)$ \\
2014 & $3.2(1.9-5.2)$ & $5.8(1.2-13.1)$ & $3.36(1.7-5.7)$ & $5.2(2.7-8.9)$ & $4.1(0.6-11.4)$ \\
2017 & $3.4(1.7-6.0)$ & $5.9(1.2-13.6)$ & $3.6(2.1-6.0)$ & $5.4(2.9-9.2)$ & $4.2(0.6-12.1)$
\end{tabular}

In 2017 the number of deaths and DALYs attributable to SHS exposure were respectively 335 (UI: 88-772) and 35,633 (UI: 11,141-78,176). The proportion of deaths and DALYs 
attributable to SHS exposure on the total number of deaths and DALYs among children in the EU was respectively $1.4 \%$ and $0.7 \%$. The proportion of DALYs varied between values higher than $1.5 \%$ in Malta, Slovakia, Bulgaria, Poland and Romania, and values lower than $0.4 \%$ in Finland, The Netherlands, Begium and Luxemburg (Figure 1).

In 2017 LBW caused most part of both deaths and years of healthy life lost due to prenatal SHS exposure, with a proportion of DALYs of $0.4 \%$ in all the EU countries, reaching a maximum of $1.1 \%$ in Poland. Low birth weight was followed by LRI, asthma, SIDS and finally by OM with around $0.1 \%$ DALYs attributable to SHS on the overall DALYs (Figure 2). In Eastern EU countries such as Poland, Bulgaria, Slovakia, and also Malta, DALYs due to SHS exposure were mainly from LBW with a proportion up to $1.1 \%$ in Poland, whereas in Romania and Bulgaria a high proportion of SHS-related DALYs was from LRI (Figure 2).

\section{Discussion}

This study provides evidence indicating a stall in SHS household exposure in children and in SHS exposure among pregnant women in all settings, and, consequently, in PAFs estimates. In the first period, from 2006 to 2010, slightly lower values of SHS exposure in children and pregnant women were estimated and this may be in part due to the implementation of smoking bans in 16 out of 28 EU countries (Luxembourg, Belgium, the UK, Spain, Finland, Slovenia, Lithuania, Denmark, France, Portugal, Croatia, Slovakia, Latvia, Cyprus, Greece, Germany). Other 6 EU countries implemented nation-wide smoking bans maximum two years before 2006, i.e. in 2004-2005 (Ireland, Sweden, Malta, Italy, Estonia, The Netherlands), whereas, after 2010, only 6 EU countries developed smoking bans (Poland, Hungary, Bulgaria, Romania, Czechia, Austria). The stall in SHS prevalence estimates from 2010 to 2017 suggests that comprehensive smoking bans had less influence in the adoption of smoke free homes in the long run. In this period, other tobacco control measures were implemented 
in EU countries with no significant impact on SHS exposure at home. Specifically, in the whole period tax increases and anti-tobacco mass-media campaigns were implemented in some countries; pictorial warnings in tobacco packages was implemented in all EU countries in 2016 and smoking bans in cars with children or pregnant women was applied in some countries in 2015-2017, e.g., in UK, France, Italy. Thus, smoking bans could have an immediate effect in slightly reducing smoking at home, but, SHS exposure still occurs and its burden is substantial in the long run.

A recent systematic review showed findings from 26 studies evaluating the burden of disease due to SHS exposure in children for different countries worldwide, with PAF estimates that largely varied among studies. PAF estimates, mainly associated to incidence, except for SIDS, ranged between $0.9 \%$ and $22.4 \%$ for OM, $6.7 \%-43.6 \%$ for SIDS, $2.0 \%-31.9 \%$ for LRI, $0.8 \%$ $35 \%$ for asthma and $2.1 \%-23.5 \%$ for LBW (3), since they are dependent on the prevalence of exposure to SHS, which largely varies across countries (25). The PAFs estimated in this study are in line with the lower ranges of those estimated in the systematic review, whereas a recent study estimated PAF attributable to SHS exposure from for OM, asthma and LRI for 2015 in Spain slightly lower in comparison to our estimates, probably due to higher a estimate of SHS exposure, defined by the existence of at least one smoker among household members (41). In 2017 the GBD study, that provides a comprehensive assessment of risk factor exposure and attributable burden of disease, estimated, for the EU countries, a total of 186 deaths and 20870 DALYs attributable to SHS exposure in children younger than 14 years of age, substantially less than our estimate of 335 deaths and 35633 DALYs (39). This is likely due to the exclusion of LBW, SIDS and asthma from the burden quantification of GBD estimates, and we showed that LBW was one of the diseases that mainly contributed to the burden from SHS exposure in children, especially in Eastern EU countries, determined from SHS exposure in pregnancy. 
Our results show that in 2017 the burden was lower in Northern European countries, such as Luxembourg, Belgium, The Netherlands, Finland, Estonia and Sweden, and higher in Eastern countries, such as Romania, Poland, Bulgaria and Slovakia. Part of the countries with a lower SHS burden are those that implemented a smoking ban earlier. Thus, the distribution of the burden due to SHS exposure by disease is not uniform in EU countries. Among those countries with a larger burden, Romania and Bulgaria showed a high proportion of DALYs attributable to SHS exposure from LRI, whereas Poland, Slovakia, Malta and Bulgaria showed a high proportion in DALYs from LBW reaching a proportion of DALYs attributable to prenatal SHS exposure on total DALYs of $1.1 \%$ in Poland. Low birth weight resulted the diseases with the highest proportion of both deaths and years of healthy life lost due to SHS exposure, suggesting a notable problem of SHS exposure in pregnancy, especially in Eastern EU countries.

There are several surveys that investigated SHS exposure in the EU countries, but SHS exposure in public places was the main studied outcome because of the evaluation of smoking bans (i.e. Global Tobacco Surveillance System and Eurobarometer surveys), whereas SHS household exposure, that is the main source of exposure in children, was not recently evaluated. The main strength of this study is that it provides a quantification of the burden due to SHS exposure at home among children in the last 10 years for the $28 \mathrm{EU}$ countries, even if the estimates were largely uncertain due to the large amount of missing values taken into account in the imputation procedure. Moreover, few studies quantified the burden form SHS exposure in pregnancy in EU or in high income countries (42), and, to our knowledge, this is the first study which provides a quantification of this burden estimating deaths and DALYs from LBW. Another strength is in the data sources: first, Eurobarometer surveys allow estimating household SHS exposure with uniform data for all the EU countries; second, mortality data for 2006, 2010, and 2014 are observed and not estimated data, and are available 
for all the diseases of interest, reducing the uncertainty that could arise from death estimates. The DALYs estimates combine an observed (years of life lost) with an estimated (years lived with disability) part, reducing the uncertainty that could arise form an estimation of the total DALYs. Moreover, another strength of this study is to use a direct question for SHS home exposure estimates, whereas, within the GBD study, household SHS prevalence was indirectly estimated using data on household composition and smoking habits (39). This study has however some limitations. A first limitation is that, given the exposure definition, changes in prevalence refer to changes in the proportion of people with children who adopt smoking rules in home, and proportion of women in fertile age being ever exposed, independently from the amount (hours) of exposure, that cannot be quantified using our data without generating further excessive uncertainty. A more precise estimation of SHS exposure taking into account for the effective quantification of exposure could improve results, but this information is not available for all the EU countries.

A second limitation comes from the age classes of SHS exposure that are not always aligned to those of the diseases under study. For example, SIDS refer to children younger than 1 year, and the burden was studied with the SHS exposure estimated for children of age 0-9 years. We can however reasonably assume that rules on smoke-free houses do not vary with the children age. Another limitation is that the quality of the meta-analysis used to select RR was not assessed, but these were chosen only on the basis of being most recent, even if some of them included old studies. A final limitation arises from the imputation procedure used to estimate SHS exposure that was used to impute data from several sample surveys by imputing the data in the combined data matrix from all surveys. This approach assumes that the relationship between SHS exposure and its explicative variables found in 2006 and 2010, remains unchanged also for years 2014 and 2017. Moreover, our imputation approach does not account for differences between the surveys, for example if they are conducted at different 
times or if different sampling methodologies or if are carried out by different organizations. A more appropriate approach is to carry out a hierarchical multiple imputation taking into account for the multilevel structure of the data given by four independent surveys $(28,31,43)$. Hierarchical multiple imputation procedures have already been developed in the literature however only take into account for multilevel continuous and binary data, and could not apply to the factor variables of our data. However, literature results show that valid inferences form hierarchal multiple imputation methods can only be obtained if the dataset gathers a large number of clusters and in our study we are working with only four clusters $(28,43)$. Moreover, the main feature of the multilevel modeling are to take into account for differences between surveys and to include covariates at survey level. In our analysis however, the pooled surveys were carried out by the same organization using the same sampling design, and the only survey level variable available was the year in which it was carried out that was included among the predictors in our imputation model (28).

In conclusion, in the last decade, despite smoke-free homes initially increased, presumably as a result of the implementation of smoking bans (44), our results show that, in the long run, the diseases caused by SHS exposure in children are still not negligible. This suggests that household SHS exposure in children and among pregnant women are still high. Up to $1.6 \%$ of total DALYs could be avoided eliminating SHS exposure in children and pregnancy . Preventing exposure of children to SHS in home and prenatal exposure to maternal SHS exposure in the EU therefore still requires great and for sure coordinated efforts.

Acknowledgements: EF thanks CERCA Programme / Generalitat de Catalunya for institutional support. 


\section{References}

1. US Department of Health and Human Services. The health consequences of involuntary exposure to tobacco smoke: a report of the Surgeon General. (US Department of Health and Human Services, Centers for Disease Control and Prevention, National Center for Chronic Disease Prevention and Health Promotion, Office on Smoking and Health, Atlanta, GA, 2006).

2. Öberg, M., Jaakkola, M.S., Woodward, A., Peruga, A., Prüss-Ustün, A. Worldwide burden of disease from exposure to second-hand smoke: a retrospective analysis of data from 192 countries. Lancet. 377, 139-46 (2011).

3. Carreras, G. et al. Burden of disease attributable to second-hand smoke exposure: A systematic review. Prev Med. 129, 105833 (2019).

4. Cheng, K.W., Glantz, S.A., Lightwood, J.M. Association between smokefree laws and voluntary smokefree-homes rules. Am J Prev Med 41, 566-572 (2011).

5. Martínez-Sánchez, J.M. et al. Do smoke-free policies in work and public places increase smoking in private venues? Tob Control.23, 204-207 (2014).

6. Monson E, Arsenault N. Effects of Enactment of Legislative (Public) Smoking Bans on Voluntary Home Smoking Restrictions: A Review. Nicotine Tob Res. 2017; 19: 141-148.

7. Minardi, V. et al. Compliance with the smoking ban in Italy 8 years after its application. Int $J$ Public Health. 59, 549-54 (2014).

8. Gallus, S. et al. Voluntary home smoking ban: prevalence, trend and determinants in Italy. Eur J Public Health. 26, 841-844 (2016).

9. Jarvis, M.J., Sims, M., Gilmore, A., Mindell, J. I mpact of smoke-free legislation on children's exposure to secondhand smoke: cotinine data from the Health Survey for England. Tob Control. 21, 18-23 (2012). 
10. Akhtar, P.C. et al. Smoking restrictions in the home and secondhand smoke exposure among primary schoolchildren before and after introduction of the Scottish smoke-free legislation. Tob Control. 18, 409-415 (2009).

11. Fernández,E. et al. Changes in Secondhand Smoke Exposure After Smoke-Free Legislation (Spain, 2006-2011). Nicotine Tob Res. 19, 1390-1394 (2017).

12. Akhtar, P.C., Currie, D.B., Currie, C.E., Haw, S.J. Changes in Child Exposure to Environmental Tobacco Smoke (CHETS) study after implementation of smoke-free legislation in Scotland: national cross sectional survey. BMJ. 335, 545 (2007).

13. Faber, T. et al. Effect of tobacco control policies on perinatal and child health: a systematic review and meta-analysis. Lancet Public Health. 2(9), e420-e437 (2017).

14. Been, J.V. et al. Smoke-free legislation and paediatric hospitalisations for acute respiratory tract infections: national quasi-experimental study with unexpected findings and important methodological implications. Tob Control. 27(e2), e160-e166 (2018).

15. Mackay, D., Haw, S., Ayres, J.G., Fischbacher, C., Pell, J.P. Smoke-free legislation and hospitalizations for childhood asthma. N Engl J Med. 363, 1139-45 (2010).

16. Hajdu, T., Hajdu, G. Smoking ban and health at birth: Evidence from Hungary. Econ Hum Biol. 30, 37-47. (2018)

17. Been, J.V., Millett, C., Lee, J.T., van Schayck, C.P., Sheikh, A. Smoke-free legislation and childhood hospitalisations for respiratory tract infections. Eur Respir J. 46, 697-706 (2015).

18. Millett, C., Lee, J.T., Laverty, A.A., Glantz, S.A., Majeed, A. Hospital admissions for childhood asthma after smoke-free legislation in England. Pediatrics.131, e495-501 (2013).

19. Martínez-Sánchez, J.M. et al. Exposure to secondhand smoke in Italian non-smokers 5 years after the Italian smoking ban. Eur J Public Health. 22, 707-12 (2012).

20. López, M.J. et al. Social inequalities in secondhand smoke exposure in children in Spain. Tob Induc Dis. 16, 14 (2018). 
21. Robinson, O. et al. The association between passive and active tobacco smoke exposure and child weight status among Spanish children. Obesity 24,1767-77 (2016)

22. Wdowiak, A., Wiktor, H., Wdowiak, L. Maternal passive smoking during pregnancy and neonatal health. Ann Agric Environ Med 16, 309-312 (2009).

23. Argalášová, L. et al. Determinants of ETS exposure in a sample of Slovak pregnant women. Rev Environ Health 32,201-205 (2017).

24. Fernández, E. et al. Tackling second-hand exposure to tobacco smoke and aerosols of electronic cigarettes: the TackSHS project protocol. Gac Sanit. 34, 77-82 (2020).

25. Öberg, M., Jaakkola, M.S., Prüss-Üstün, A., Schweizer, C., Woodward, A. Second-hand smoke: Assessing the environmental burden of disease at national and local levels. (World Health Organization, WHO Environmental Burden of Disease Series, No. 18, Geneva, 2010).

26. Levin, M. The occurrence of lung cancer in man. Acta Unio Int. Contra Cancrum. 9, 531-541 (1953).

27. Rubin, D.B. Multiple Imputation for Nonresponse in Surveys. (John Wiley \& Sons, New York, 1987).

28. Gelman, A., King, G., Liu, C. Not asked and not answered: multiple imputation for multiple surveys. JASA 93, 846-857 (1999).

29. White, I.R., Royston, P., Wood, A.M. Multiple imputation using chained equations: Issues and guidance for practice. Stat Med. 30, 377-99 (2011).

30. Raghunathan, T.E., Lepkowski, J.M., van Hoewyk, J., Solenberger, P. A multivariate technique for multiply imputing missing values using a sequence of regression models. Survey Methodology. 27, 85-95 (2001).

31. Resche-Rigon, M., White, I.R. Multiple imputation by chained equations for systematically and sporadically missing multilevel data. Stat Methods Med Res. 27, 1634-1649 (2018). 
32. Eurostat. Fertility statistics. Mean age of women at childbirth across EU regions. https://ec.europa.eu/eurostat/statistics-explained/index.php/Fertility_statistics (accessed 29/09/2020)

33. Leonardi-Bee, J., Smyth, A., Britton, J., Coleman, T. Environmental tobacco smoke and fetal health: systematic review and meta-analysis. Arch Dis Child Fetal Neonatal Ed. 93, F351-61 (2008).

34. Royal College of Physicians. Passive smoking and children. A report by the Tobacco Advisory Group. (RCP, London, 2010).

35. Jones, L. et al. Parental and household smoking and the increased risk of bronchitis, bronchiolitis and other lower respiratory infection in infancy: systematic review and metaanalysis. Respir Res. 12:5 (2011).

36. Li, J.S., Peat, J.K., Xuan, W., Berry, G. Meta-analysis on the association between environmental tobacco smoke (ETS) exposure and the prevalence of lower respiratory tract infection in early childhood. Pediatr Pulmonol 27: 5-13 (1999).

37. Tinuoye, O., Pell, J.P., Mackay, D. Meta-analysis of the association between secondhand smoke exposure and physician-diagnosed childhood asthma. Nicotine Tob Res. 15, 1475-83 (2013).

38. Jones, L., Hassanien, A., Cook, D., Britton, J., Leonardi-Bee, J. Parental smoking and the risk of middle ear disease in children. Arch Pediatr Adolesc Med. 166, 18-27 (2012).

39. GBD 2016 Risk Factors Collaborators. Global, regional, and national comparative risk assessment of 84 behavioural, environmental and occupational, and metabolic risks or clusters of risks, 1990-2016: a systematic analysis for the Global Burden of Disease Study 2016. Lancet. 390(10100), 1345-1422 (2017).

40. Stevens, G.A. et al. Guidelines for Accurate and Transparent Health Estimates Reporting: the GATHER statement. Lancet. 388, e19-23 (2016). 
41. Continente, X. et al. Burden of respiratory disease attributable to secondhand smoke exposure at home in children in Spain (2015). Prev Med. 123:34-40 (2019).

42. Reece, S. et al. Secondhand smoke exposure during pregnancy: a cross-sectional analysis of data from Demographic and Health Survey from 30 low-income and middle-income countries. Tob Control 28, 420-426 (2019).

43. Audigier, V. et al. Multiple imputation for multilevel data with continuous and binary variables. Statistical Science. 33, 160-183 (2018).

44. Behbod, B., Sharma, M., Baxi, R., Roseby, R., Webster, P. Family and carer smoking control programmes for reducing children's exposure to environmental tobacco smoke. Cochrane Database Syst Rev. 1, CD001746 (2018). 
Figure 1 - Rank of the proportion of disability-adjusted life years (DALYs) attributable to second-hand smoke on total DALYs among children in 2017 in the European Union. AT: Austria, BE: Belgium, BG: Bulgaria, CY: Cyprus, CZ: Czech Republic, HR: Croatia, DK: Denmark, EE: Estonia, FI: Finland, FR: France, DE: Germany, GR: Greece, HU: Hungary, IE: Ireland, IT: Italy, LV: Latvia, LT: Lithuania, LU: Luxembourg, MT: Malta, NL:

Netherlands, PL: Poland, PT: Portugal, RO: Romania, SK: Slovakia, SI: Slovenia, ES: Spain, SE: Sweden, UK: United Kingdom.

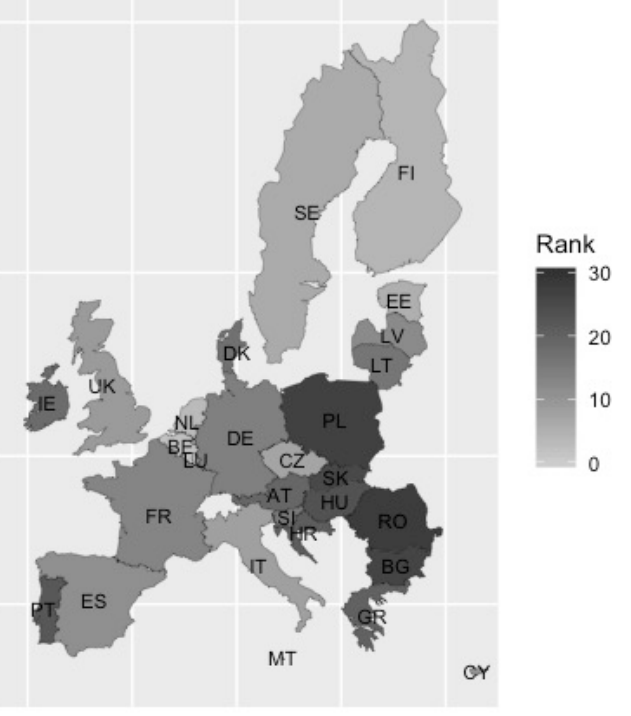

Figure 2 - Proportion of DALYs attributable to SHS on total DALYs by cause and Country, 2017.

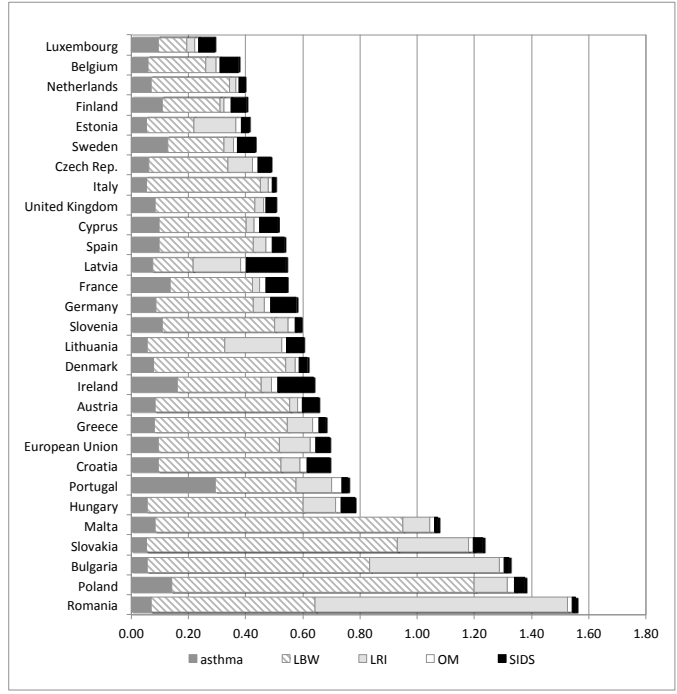

